\title{
Anomalous electrostatic potential properties in CNT thin films under a weak external electric field
}

\author{
U Ishiyama ${ }^{1}$, Nguyen Thanh Cuong ${ }^{2}$, and Susumu Okada ${ }^{1}$ \\ ${ }^{1}$ Graduate School of Pure and Applied Sciences, University of Tsukuba, Tsukuba, Ibaraki 305-8571, Japan \\ ${ }^{2}$ International Center for Young Scientist (ICYS) and International Center for Materials Nanoarchitectonics \\ (MANA), National Institute for Materials Science (NIMS), Tsukuba, Ibaraki 305-0044, Japan
}

\begin{abstract}
Based on density functional theory, we studied electronic properties of carbon nanotube (CNT) thin films under an electric field. The carrier accumulation due to the electric field strongly depends on the CNT species forming thin films. For the low electron concentration, we find that the injected electrons are extended throughout the CNTs, leading to the unusual electric filed between CNTs of which direction is opposite to the applied field. This unusual field response of CNT thin films on the external electric field is ascribed to the internal electric field arising from the electrostatic potential difference between constituent CNTs.
\end{abstract}

Following the discovery of carbon nanotubes $(\mathrm{CNT})^{1)}$ in soot, CNTs have attracted much attention because of their unique geometric and electronic structures, which have extended fields of low-dimensional sciences and technologies. The electronic structures of CNTs are metallic, semiconducting, or magnetic, depending on the boundary conditions imposed on their honeycomb networks along their circumference and length. ${ }^{2-6)}$ For the mechanical aspect, CNTs exhibit high Young's modulus ${ }^{7-10)}$ and remarkable thermal conductivity ${ }^{11-13)}$ arising from their rigid covalent networks of $s p^{2} \mathrm{C}$ atoms. In addition, the nanoscale tubular space of the CNT can accommodate foreign atoms and molecules, resulting in the interesting hybrid systems whose physical/chemical properties are different from the simple sum of each constituent unit. ${ }^{14-16)}$ These unusual and fascinating physical properties allow them to be premier materials in wide areas of modern technologies, such as electronic, mechanical, thermal, and energy devices. Among these wide possible applications, it has been demonstrated that CNTs work as conducting channel of field effect transistors (FETs) ${ }^{17-21,24)}$ and via interconnects of semiconductor electronic devices. ${ }^{22,23)}$

In usual CNT-based FETs, CNTs have mat film structures in which CNTs intrinsically possess intersections with the other CNTs. At the intersectional regions, CNTs form a stacking or multi-decker structures with respect to the gate electrode. Although, the CNT mat films can work as conducting channel in real devices such as multi-stage ring oscillators, ${ }^{21,24)}$ it is still unclear a microscopic mechanism of the carrier accumulation in CNT mat films by a gate electrodes. In particular, it is uncertain whether the nanoscale interfaces in CNT 
thin films and the inhomogeneity of the CNT species affect the carrier accumulations by the gate electrode in FET structures or not. Thus, in this manuscript, we aim to elucidate the fundamental electronic properties of CNT thin films, which are simulated by the bilayer of CNTs with different diameters, under an external electric field which injects electrons/holes into the CNT thin films within a framework of the density functional theory. Our calculations show that the carrier accumulation due to the electric field strongly depends on the field strength and the CNT species comprising the thin films. For the high carrier concentration or large gate voltage, the carriers are primary distributed on the CNT wall at the electrode side. In contrast, the injected electrons are extended throughout the CNTs for the low electron concentrations or the weak positive gate voltage. The extended electrons cause the unusual electric filed between CNTs with particular arrangements, of which direction is opposite to the applied field by the gate electrode. Detailed analysis of the electrostatic potential of the thin films clarified that the unusual field dependence of the CNT thin films under the low electron concentration is ascribed to the internal electric field arising from the work function difference between constituent CNTs.

All calculations were performed within the framework of the density functional theory $(\mathrm{DFT})^{25,26)}$ using a STATE package. ${ }^{27)}$ To express the exchange correlation potential among interacting electrons, we use the local density approximation with a functional form fitted to Monte-Carlo results for homogeneous electron gas generated by Perdew and Wang. ${ }^{28,29)} \mathrm{We}$ use an ultrasoft pseudopotential to describe the interactions between the valence electrons and ions generated by the Vanderbilt scheme. ${ }^{30)}$ The valence wave function and charge density were expanded in terms of the plane-wave basis set with cutoff energies of 25 and $225 \mathrm{Ry}$, respectively. Integration over one-dimensional Brillouin zone is carried out using 4 equidistance $k$ points for the single periodicity of zigzag CNTs. We adopt the effective screening medium $(\mathrm{ESM})^{31)}$ method to solve the Poisson equation including the excess electrons or holes injected by the electrode within the framework of the DFT using the plane-wave basis set.

To simulate the intersection of CNTs in FET devices under the gate voltage, we consider a structural model shown in Fig. 1, in which parallelly arranged two CNTs are located below the planar gate electrode simulated by an effective screening medium to mimic an ideal metal electrode. For CNT thin films, we consider semiconducting (11,0), (13,0), and (14,0) CNTs with the arrangements of CNT1-CNT2 where CNT1 is located at the electrode side with the vacuum spacing of about $9 \AA$ while CNT2 is located below the CNT1 by the spacing of 3.3 $\AA$. Since the model is asymmetric with respect to the electrode, we consider the following CNT thin films consisting of pairs of $(11,0),(13,0)$, and $(14,0)$ CNTs. An electric field is applied between the electrode and the CNTs up to about $\mathrm{V}_{G}= \pm 5 \mathrm{~V}$, corresponding to 0.3 electrons/holes injection into CNTs. The atomic structures were optimized until the force acting on each atom is less than $5 \mathrm{meV} / \AA$ under the fixed lattice parameters for the tube 


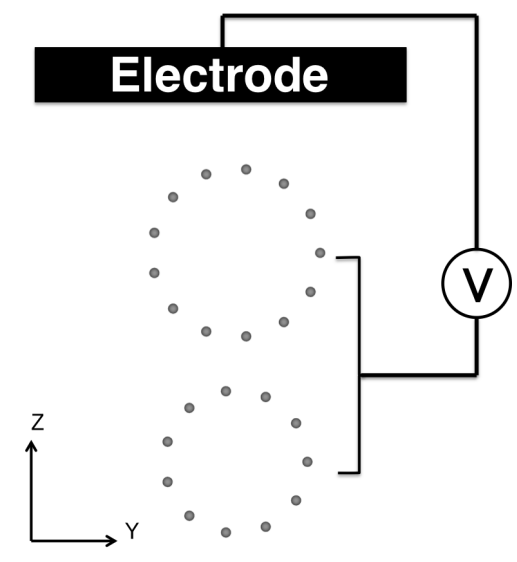

Fig. 1. A structural model of CNT-FETs consisting of a bilayer of CNT1 and CNT2. Gray circles denote the atomic position of the CNTs

axis of $4.2 \AA$. During the calculations under the conditions with a finite electric field, the geometries were kept the same as those with a zero electric field.

Figure 2 shows the distribution of accumulated carriers in CNT thin films under the electric field. In all cases, accumulated carriers, both electrons and holes, are mainly distributed at the electrode side of the CNT. However, for the electron concentration of 0.1e, CNT thin films consisting of $(11,0),(13,0)$, and $(14,0)$ CNTs exhibit unusual distribution of accumulated carriers within and between CNTs: The accumulated carriers are extended throughout CNT1 located at the electrode side compared with the cases of the high electron concentration and of hole doping. In addition to the extended carriers in the CNT1, carriers are induced on the wall of the CNT2 located at the opposite side to the electrode. Distributions of induced carriers depends on the CNT species and their relative arrangement to the electrode. For the $(13,0)-(11,0)$ and $(13,0)-(14,0)$ thin films, holes are induced at the opposite side of $(13,0) \mathrm{CNT}$ to the electrode, so that the electrons are accumulated in the wall of $(11,0)$ and $(14,0)$ CNTs in the second CNT layer. In contrast, for the $(11,0)-(13,0)$ and $(14,0)-(13,0)$ thin films, the electrons are induced at the opposite side of $(11,0)$ and $(14,0)$ CNTs to the electrode, so that the holes are induced into the $(13,0)$ CNT.

Figure 3 shows the contour plots of the electrostatic potential and the vector plots of the electric field of CNT thin films under the electric field. Electric field or the potential gradient mainly emerges between the wall of CNT1 and electrode in accordance with the accumulated carrier by the electric field. The fact indicates that the electrostatic potential modulation in CNT thin films under the carrier accumulation by the external electric field is mostly screened by the CNT in the first layer. By focusing on the detailed electric field between CNTs under the low electron concentration, we find unusual field properties in the $(11,0)-(13,0)$ and $(14,0)$ $(13,0)$ thin films. Accumulated carriers between CNTs cause the over screening against the 
(a)
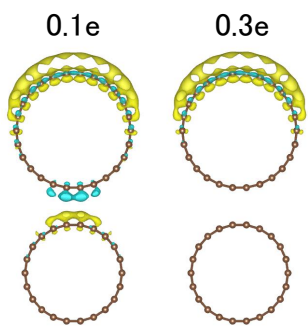

$\sum_{0,000}^{000}$ \$

(c)
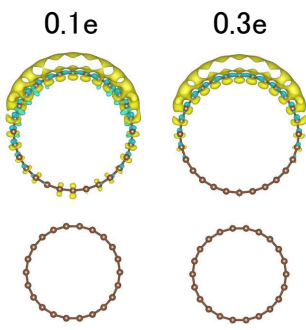

(e)

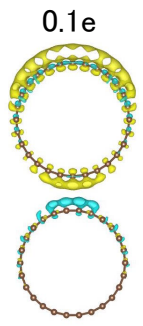

c)
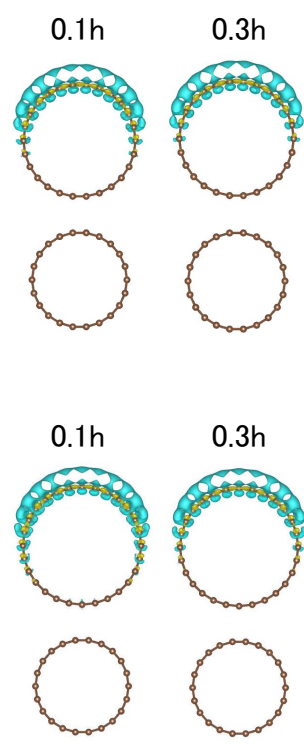

$0.3 \mathrm{e}$
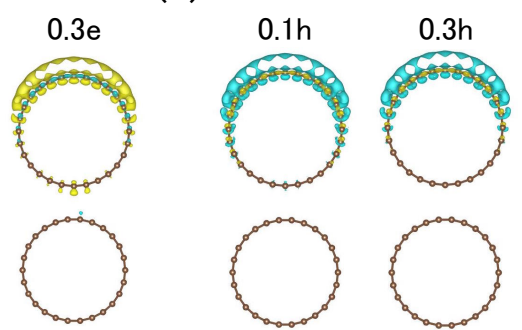
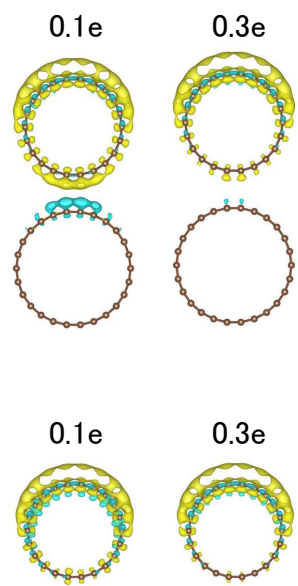

(b)
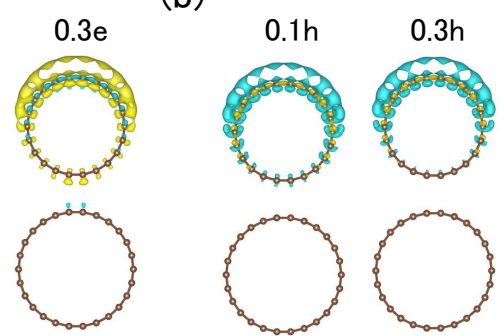

(d)
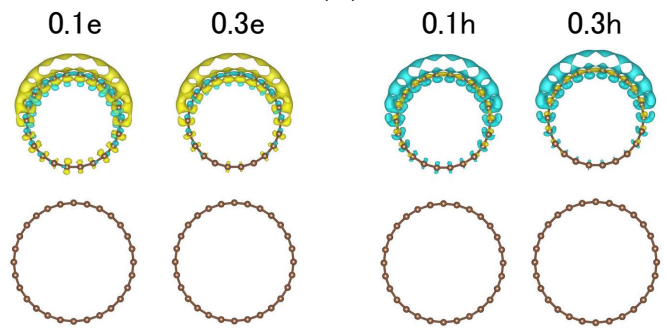

(f)
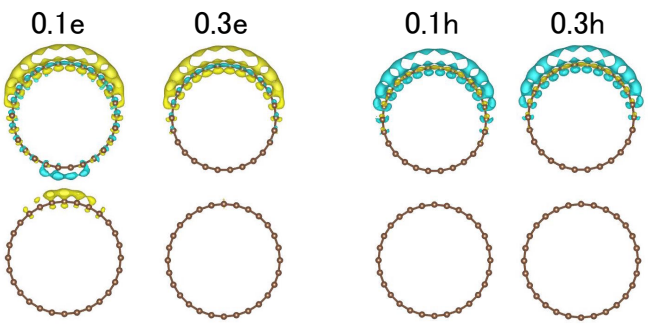

Fig. 2. (Color online) Isosurfaces of the accumulated and depressed carriers in CNT thin films of (a) $(13,0)-(11,0)$ CNTs, (b) $(11,0)-(13,0)$ CNTs, (c) $(14,0)-(11,0)$ CNTs, (d) $(11,0)-(14,0)$ CNTs, (e) $(14,0)-(13,0) \mathrm{CNTs}$, and (f) $(13,0)-(14,0)$ CNTs by an external electric field. Yellow and cyan isosurfaces indicate the region where electron and hole increase, respectively, under the electric field.

applied external electric field, leading to the weak opposite electric field to the external field. Thus, under the low electron concentration, CNT thin films may exhibit usual response on the external electric field, which causes the variation in device properties of CNT thin film transistors.

To investing the physical origin of the unusual carrier accumulation in CNT thin films for the low electron concentration, we depict the plane-averaged electrostatic potential of CNT thin films along the z-direction under a zero electric field (Fig. 4). The electrostatic potential at the left cell boundary is not identical to that at the right cell boundary, indicating that the intrinsic potential difference between CNTs causes the electric field between them. The internal electric field exhibits the symmetric nature with respect to CNT replacement with respect to the electrodes as listed in Table I. Furthermore, the potential difference strongly depends on the CNT species. A negative electric field of $65 \mathrm{mV} / \mathrm{nm}$ is induced for the $(13,0)$ - 
(a)
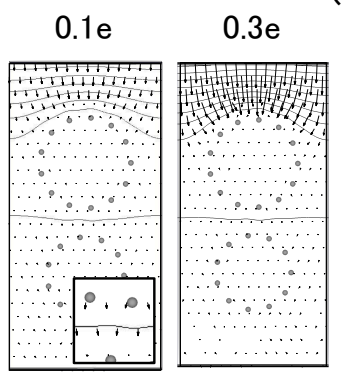

(c)
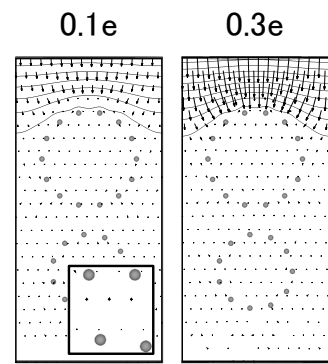

(e)

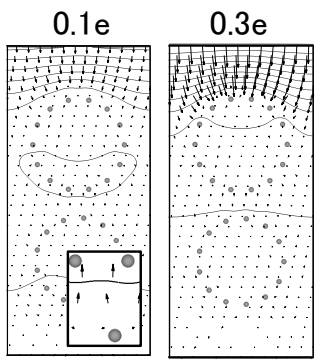

$0.1 \mathrm{~h}$
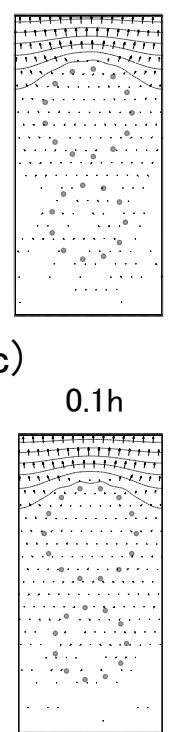

e)
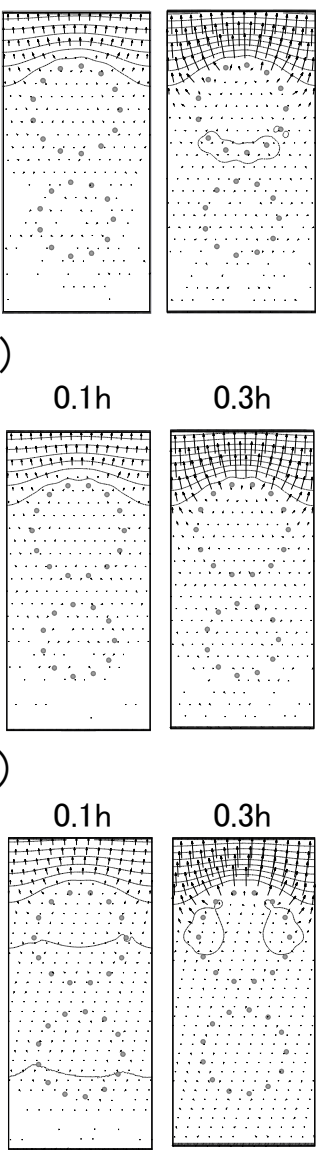

$0.3 \mathrm{~h}$

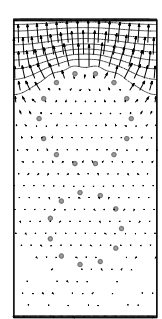

(b)
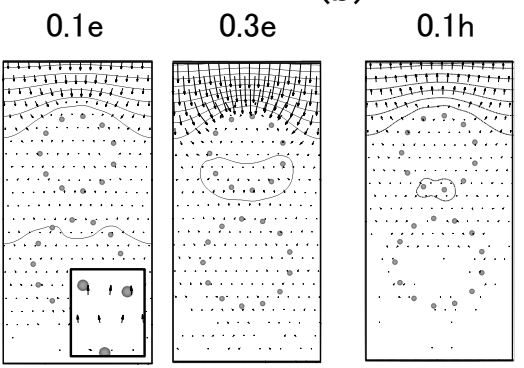

$0.3 \mathrm{~h}$
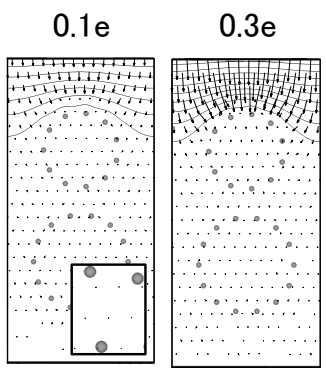

(d)

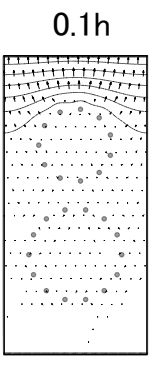

(f)

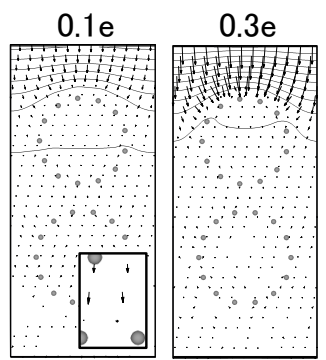

$0.3 \mathrm{~h}$
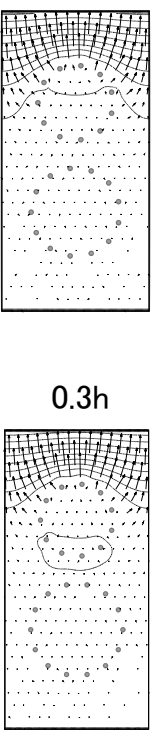

$0.3 \mathrm{~h}$

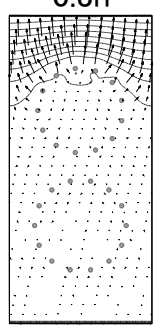

Fig. 3. Contour plots of the electrostatic potential and the vector plots of the electric field of (a) (13,0)-(11,0), (b) (11,0)-(13,0), (c) $(14,0)-(11,0)$, (d) $(11,0)-(14,0),(e)(14,0)-(13,0)$, and (f) (13,0)$(14,0)$ CNT thin films with excess electrons (0.1e and 0.3e) and holes (0.1h and 0.3h). Gray circles denote the atomic position of CNTs. Insets in the leftmost panel in each figure denote the enlarged picture of the potential and the electric field at CNT interfaces.

$(11,0)$ thin film, while the positive electric field of $64 \mathrm{~m} / \mathrm{nm}$ is induced for the $(11,0)-(13,0)$ thin film. The field is $9 \mathrm{mV} / \mathrm{nm}$ for the thin film consisting of $(14,0)$ and $(11,0)$ CNTs, which is smaller than that for the thin film consisting of $(11,0)$ and $(13,0)$ CNTs. The field direction in $(11,0)-(13,0)$ thin film is opposite to that of the $(13,0)-(11,0)$ thin film. The field is $54 \mathrm{mV} / \mathrm{nm}$ for the $(14,0)-(13,0)$ thin film, while the field is $-55 \mathrm{mV} / \mathrm{nm}$ for the $(13,0)-(14,0)$ thin film. The fact indicates that the CNT thin films comprising the mixture of CNT with the different family pattern (a $(n, m) \mathrm{CNT}$ with the index $2 n+m=3 k+1$ and that with the index of $2 n+m=3 k+2$ where the $k$ is the integer) have large inter-CNT electric field, leading to the unusual internal electric field against the weak external electric field. Thus, the internal electric field in CNT thin films causes the unusual carrier accumulation in CNT thin films by 

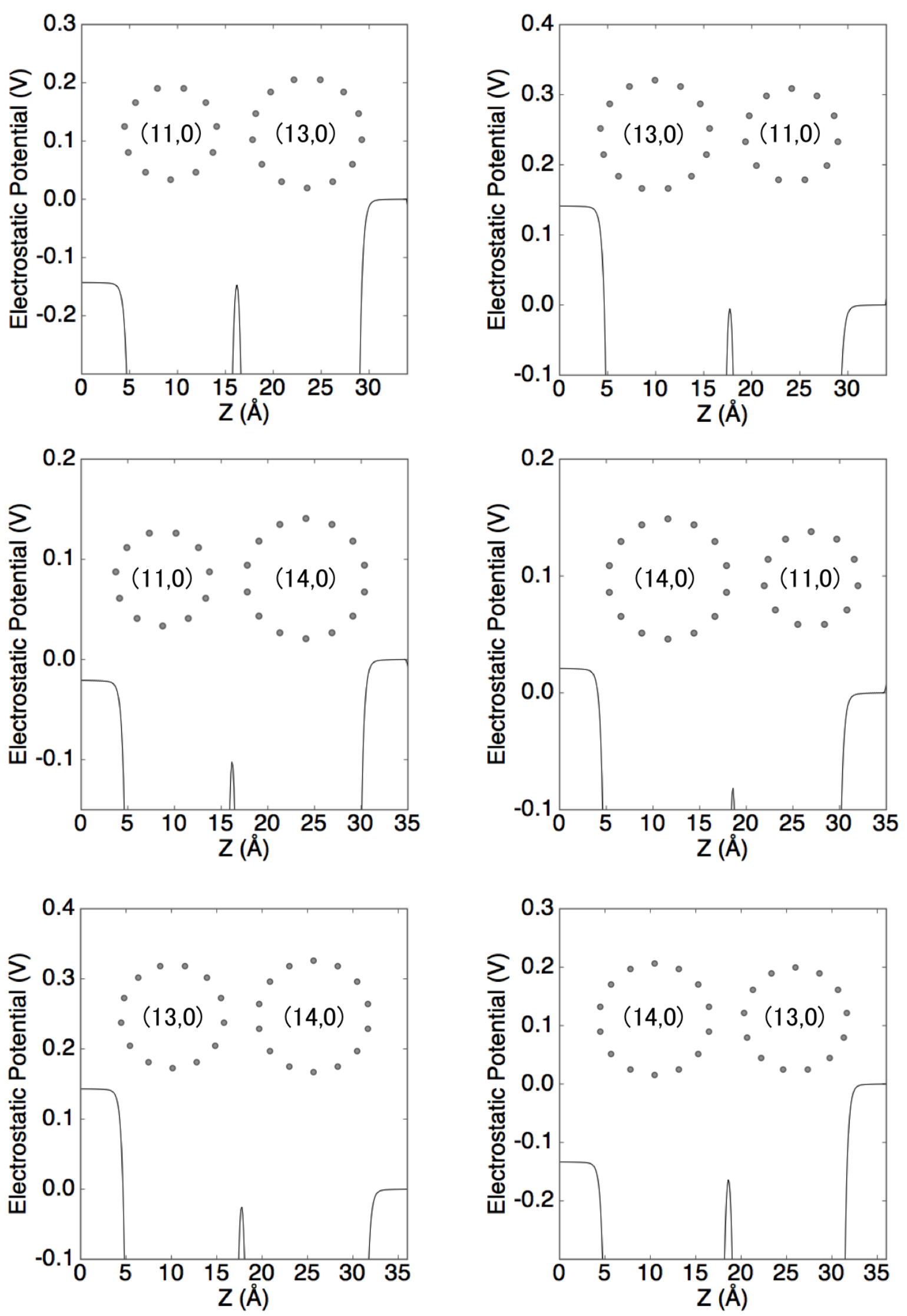

Fig. 4. Plane averaged electrostatic potential of CNT thin films normal to the z-direction under zero electric field. The electrode is located at the right end of each panel. 
Table I. Intrinsic electric field between CNTs in the thin films $(\mathrm{mV} / \mathrm{nm})$. CNT1

\begin{tabular}{ccccc} 
& & $(11,0)$ & $(13,0)$ & $(14,0)$ \\
\hline & $(11,0)$ & - & -65.45 & -9.25 \\
CNT2 & $(13,0)$ & 64.53 & - & 54.02 \\
& $(14,0)$ & 9.16 & -55.26 & - \\
\hline
\end{tabular}

the gate electrode.

In summary, we studied the electronic structures of CNT thin films, simulated by the parallelly aligned CNTs, under the carrier accumulation by the gate electrode using the density functional theory by combining with the effective screening medium method. Our calculations show that the carrier accumulation due to the electric field strongly depends on the CNT species comprising the thin films, their arrangement with respect to the electrode, and the carrier concentration. For the high carrier concentration or large gate voltage, the carriers are primary distributed on the CNT wall at the electrode side. In contrast, the injected electrons are extended throughout the CNTs for the low electron concentrations or the weak positive gate voltage. By investing the electrostatic potential and the electric field, we find that the electric field induced by the gate electrode is mostly screened in the first layer of CNTs located at the electrode side, due to the strongly localized nature of the accumulated carriers. On the other hand, for the low electron concentration, the extended nature of the accumulated carriers causes the unusual electric filed between CNTs with particular CNT arrangements, of which direction is opposite to the applied field by the gate electrode. Detailed analysis of the electrostatic potential of the thin films clarified that the unusual field dependence is ascribed to the internal electric field arising from the work function difference between constituent CNTs, which depend not only on diameter of CNT but also on the family pattern.

Acknowledgment This work was supported in part by a Grant-in-Aid for Scientific Research from the Ministry of Education, Culture, Sports, Science and Technology of Japan and the Joint Research Program on Zero-Emission Energy Research, Institute of Advanced Energy, Kyoto University. Computations were performed on a NEC SX-8/4B at the University of Tsukuba, a SGI ICE XA/UV at the Institute for Solid State Physics, The University of Tokyo, and a NEC SX-Ace at the Cybermedia Center, Osaka University. 


\section{References}

1) S. Iijima, Nature, 354, 56 (1991).

2) N. Hamada, S.-I. Sawada, and A. Oshiyama, Phys. Rev. Lett. 68, 1579 (1992).

3) R. Saito, M. Fujita, M. S. Dresselhaus, and G. Dresselhaus, Appl. Phys. Lett. 60, 2204 (1992).

4) K. Tanaka, K. Okahara, M. Okada, and T. Yamabe, Chem. Phys. Lett. 191, 469 (1992).

5) S. Okada and A. Oshiyama, J. Phys. Soc. Jpn. 72, 1510 (2003).

6) S. Okada, NANO 2, 51 (2007).

7) R. S. Ruoff and D. C. Lorents, Carbon 33, 925 (1995).

8) M. M. J. Treacy, T. W. Ebbesen, and J. M. Gibson, Nature, 381, 678 (1996).

9) M.-F. Yu, O. Lourie, M. J. Dyer, K. Moloni, T. F. Kelly, and R. S. Ruoff, Science 287, 637 (2000).

10) M.-F. Yu, B. S. Files, S. Arepalli, and R. S. Ruoff, Phys. Rev. Lett. 84, 5552 (2000).

11) S. Berber, Y.-K. Kwon, and D. Tománek, Phys. Rev. Lett. 84, 4613 (2000).

12) T. Yamamoto, S. Watanabe, and K. Watanabe, Phys. Rev. Lett. 92, 075502 (2004).

13) T. Yamamoto and K. Watanabe, Phys. Rev. Lett. 96, 255503 (2006).

14) B. W. Smith, M. Monthioux, and D. E. Luzzi, Nature, 396, 323 (1998).

15) S. Okada, S. Saito, and A. Oshiyama, Phys. Rev. Lett. 86, 3835 (2001).

16) M. Monthioux, Carbon 40, 1809 (2002).

17) S. J. Tans, A. R. M. Verschueren, and C. Dekker, Nature 393, 49 (1998).

18) R. Martel, T. Schmidt, H. R. Shea, T. Hartel, and Ph. Avouris, Appl. Phys. Lett. 73, 2447 (1998).

19) Y. Nosho, Y. Ohno, S. Kishimoto, and T. Mizutani, Appl. Phys. Lett. 86, 073105 (2005).

20) Y. Nosho, Y. Ohno, S. Kishimoto, and T. Mizutani, Nanotechnology 17, 3412 (2006).

21) D.-M. Sun, M. Y. Timmermans, Y. Tian, A. G. Nasibulin, E. I. Kauppinen, S. Kishimoto, T. Mizutani, and Y. Ohno, Nat. Nanotechnol. 6, 156 (2011).

22) M. Nihei, D. Kondo, A. Kawabata, S. Sato, H. Shioya, M. Sakaue, T. Iwai, M. Ohfuti, and Y. Awano, IEEE International Interconnect Technology Conference, Tech. Dig., 2005, p.234-236.

23) S. Sato M. Nihei, A. Mimura, A. Kawabata, D. Kondo, H. Shioya, T. Iwai, M. Mishima, M. Ohniti, and Y. Awano, IEEE International Interconnect Technology Conference, Tech. Dig., 2006, p.230-232. 
24) D.-M. Sun, M. Y. Timmermans, A. Kaskela, A. G. Nasibulin, S. Kishimoto, T. Mizutani, E. I. Kauppinen, and Y. Ohno, Nature Commun. 4, 2302-1-8 (2013).

25) P. Hohenberg and W. Kohn, Phys. Rev. 136, B864 (1964).

26) W. Kohn and L. J. Sham, Phys. Rev. 140, A1133 (1965).

27) Y. Morikawa, K. Iwata, and K. Terakura, Appl. Surf. Sci. 169-170, 11 (2000).

28) J. P. Perdew and A. Zunger, Phys. Rev. B 23, 5048 (1981).

29) D. M. Ceperley and B. J. Alder, Phys. Rev. Lett. 45, 566 (1980).

30) D. Vanderbilt, Phys. Rev. B 41, 7892 (1990).

31) M. Otani and O. Sugino, Phys. Rev. B 73, 115407 (2006). 